\title{
Assessing of Depression Status and Influencing Factors Were Associated with Hospitalized Patients with 6 Chronic Diseases
}

\author{
Yanfang Deng ${ }^{1}$, Jinglan Luo ${ }^{2, *}$, Ping $\mathrm{Li}^{3}$ \\ ${ }^{1}$ Psychiatry Department, The First Affiliated Hospital of Jinan University, Guangzhou, China \\ ${ }^{2}$ Internal Medicine Department, The First Affiliated Hospital of Jinan University, Guangzhou, China \\ ${ }^{3}$ Cardiovascular Medicine Department, The First Affiliated Hospital of Jinan University, Guangzhou, China
}

Email address:

804434822@qq.com (Yanfang Deng), tlj1@jnu.edu.cn (Jinglan Luo),617507271@qq.com (Ping Li)

${ }^{*}$ Corresponding author

\section{To cite this article:}

Yanfang Deng, Jinglan Luo, Ping Li. Assessing of Depression Status and Influencing Factors Were Associated with Hospitalized Patients with 6 Chronic Diseases. American Journal of Psychiatry and Neuroscience. Vol. 8, No. 2, 2020, pp. 26-29. doi: 10.11648/j.ajpn.20200802.11

Received: April 8, 2020; Accepted: April 23, 2020; Published: June 4, 2020

\begin{abstract}
Objective: To assess depression status and influencing factors were associated with hospitalized patients with 6 chronic diseases. Methods: We collect the data from 485 patients, they agree to join our study. They were diagnosed as one of 6 chronic diseases, such as stroke, coronary heart disease, chronic obstructive pulmonary disease, diabetes, malignant tumor and chronic kidney disease. The diagnosis of time is from January 2018 to July 2018. Additionally, we collect the information by questionnaires, the information includes patient basic information, patient business situation and depression status. The questionnaires include Self-made patients general information questionnaire and patient health questionnaire (PHQ-9) which is base on the Diagnostic and Statistical Manual of Mental Disorders (DSM-IV). Result: Base on results of demographic data analysis, 205 out of 485 patients with chronic diseases had depressive symptoms. The incidence of depressive symptoms was $42.3 \%$, that include 101 cases $(49.3 \%)$ of mild depression, 62 cases $(30.2 \%)$ of moderate depression and $42(20.5 \%)$ cases of major depression. In research of depressive symptoms in patients with chronic diseases, those data show statistical significance in demographic analysis as independent variables, such as education level, average monthly medical expenses, duration of illness, number of hospitalizations and self-care ability. Conclusion: On the hand, the course of the disease is positively correlated with the incidence of depressive symptoms, the longer the course the higher the incidence of depression, consistent with similar studies. On another hand, the worse the self-care ability, the higher the incidence of depression, consistent with the results of similar studies.
\end{abstract}

Keywords: Chronic Diseases, Depression, Mental Health

\section{Introduction}

Depression and related mental disorders had strongly influence to individuals, businesses, and societies, it exact an enormous toll to them [1]. In National Health and Nutrition Examination Survey, the report shown $8 \%$ of the adult population experienced depression in a 2-week period. Additionally, $80 \%$ of those individuals reporting some difficulty with work, home and social activities because of depression [2, 3]. Base on assessment of American Psychiatric Association, the total economic burden of Major Depressive Disorder was 210.5 billion dollars per year in the United
States, with an additional 1.90 dollars spent on Major Depressive Disorder-related indirect costs for every dollar pent directly on MDD in 2010 [4]. In addition, workers with depression reported almost four times as much lost productive time at work as those without depression [5].

In China, a report shown the incidences of chronic diseases are increasing rapidly, the chronic diseases complicated with frequent occurrence of depression and other emotional problems [6]. But those depression and other emotional problems are often underestimated in some report [7]. Base on the report, the chronic diseases and emotional problems can influence patients' quality of life in interaction effect [8]. In 
previous studies, the chronic diseases and co-morbid emotional problems have focused more on a single disease, rare studies have examined the common influencing factors of depressive emotions in multiple diseases [9, 10]. The aim of this study is assessing depression status and influencing factors were associated with hospitalized patients with 6 chronic diseases, the 6 chronic diseases included coronary heart disease, stroke, diabetes, malignant tumor, chronic obstructive pulmonary disease and chronic kidney disease.

\section{Methods}

\subsection{Participants Enrollment and Survey Methods}

We invest 500 patients to join our study, but only 489 patients agree to join our study, 11 patients were excluded. Unfortunately, only 485 (97\%) questionnaires are conforming to standard. They were diagnosed as one of 6 chronic diseases, that include stroke, coronary heart disease, chronic obstructive pulmonary disease, diabetes, malignant tumor and chronic kidney disease. The diagnosis of time is from January 2018 to July 2018.

Moreover, the patients who met the inclusive criteria were investigated by questionnaire survey. Before the survey, the members of the survey team were trained collectively. The questionnaire was pre-investigated in a small area of the tertiary hospital and then modified before determination. Older patients and those who could not write were surveyed one-to-one; the rest were guided to fill out the questionnaire either electronically or in print. All data were checked and collected on-site.

The research information includes patient basic information, patient business situation and depression status. The questionnaires include Self-made patients general information questionnaire and patient health questionnaire (PHQ-9) which is base on the Diagnostic and Statistical Manual of Mental Disorders (DSM-IV) [11].

Their inclusion criteria were: (1) the patients were diagnosed as one of 6 chronic diseases; (2) They had depression; (3) Patients volunteered to participate the study. Their withdraw criteria were: (1) Inability to communicate effectively and Severe mental illness; (2) The illness status is too serious.

\subsection{Statistical Methods}

SPSS19.0 software was employed for statistical analysis. Descriptive statistics were used to analyze the general demographic characteristics of the subjects and the incidence of depression. The influence of demographic data on depressive symptoms was analyzed by the methods of chi-square test; Logistic Regression was used to analyze and screen the influencing factors. The test level: $\alpha=0.05, \quad \mathrm{P}<0.05$ was considered statistically significant.

\section{Result}

This study included 485 patients with chronic diseases, the collected demographic data include sex, age, education level, income, average monthly medical expenses, course of the disease, number of hospitalizations and self-care ability (Table 1). They were classified by frequency and constitution ratio method.

Table 1. Demographic data of patients with 6 chronic diseases $(n=485)$.

\begin{tabular}{llll}
\hline Variables & Groups & n & \% \\
\hline Sex & Male & 310 & 63.9 \\
& $<45$ & 51 & 10.5 \\
Age (Year) & $45 \sim$ & 134 & 27.6 \\
& $60 \sim$ & 223 & 46.0 \\
& $\geq 75$ & 77 & 15.9 \\
Education level & Primary School & 182 & 37.5 \\
& Middle School & 109 & 22.5 \\
& Technical Secondary / High School & 21.0 \\
Family monthly income (CNY) & College and above & 102 & 19 \\
& $<2000$ & 92 & 7.4 \\
Average monthly medical expenses (CNY) & $2000 \sim$ & 36 & 12.6 \\
& $5000 \sim$ & 61 & 40.6 \\
Family company & $10000 \sim$ & 197 & 39.4 \\
& $<2000$ & 191 & 61 \\
Complications (Species) & $2000 \sim$ & 296 & 14.6 \\
& $5000 \sim$ & 71 & 10.7 \\
& $10000 \sim$ & 52 & 13.6 \\
Course of the disease (Year) & Yes & 66 & 90.1 \\
& 0 & 441 & 80.6 \\
Number of hospitalizations (Time) & 1 & 391 & 15.9 \\
\hline & 2 & 94 & 2.5 \\
& $3 \sim$ & 77 & 1.0 \\
& $<1$ & 5 & 32.8 \\
& $1 \sim$ & 159 & 34.2 \\
& $10 \sim$ & 166 & 33.0 \\
& 1 & 160 & 28.2 \\
& $2 \sim$ & 137 & 71.8 \\
\end{tabular}




\begin{tabular}{llll}
\hline Variables & Groups & n & \% \\
\hline & Completely Self-sufficient & 296 & 61.0 \\
Self-care ability & Partial Self-sufficient & 143 & 29.5 \\
& In Need of Great Help & 35 & 7.2 \\
& Totally Dependent & 11 & 2.3 \\
\hline
\end{tabular}

The results of demographic data analysis showed that there were significant differences in education level, course of the disease, average monthly medical expenses and self-care ability between depression group and non-depression Group (Table 2). 205 out of
485 patients with chronic diseases had depressive symptoms. The incidence of depressive symptoms was $42.3 \%$, that include 101 cases $(49.3 \%)$ of mild depression, 62 cases $(30.2 \%)$ of moderate depression and $42(20.5 \%)$ cases of major depression.

Table 2. Comparison of demographic data between depression group and non-depression Group ( $n=485$ ).

\begin{tabular}{|c|c|c|c|c|c|}
\hline \multirow{2}{*}{$\begin{array}{l}\text { Variables } \\
\text { Sex }\end{array}$} & & \multirow[t]{2}{*}{ With symptoms } & \multirow[t]{2}{*}{ Without symptoms } & \multirow{2}{*}{$\begin{array}{l}\mathbf{X}^{2} \text { value } \\
0.697\end{array}$} & \multirow{2}{*}{$\begin{array}{l}\boldsymbol{P} \text {-value } \\
0.069\end{array}$} \\
\hline & & & & & \\
\hline \multirow{6}{*}{ Age (Year) } & Male & 129 & 181 & & \\
\hline & Female & 76 & 99 & & \\
\hline & $<45$ & 25 & 26 & 7.098 & 0.069 \\
\hline & $45 \sim$ & 56 & 78 & & \\
\hline & $60 \sim$ & 83 & 140 & & \\
\hline & $\geq 75$ & 41 & 36 & & \\
\hline \multirow{5}{*}{ Education level } & & & & 0.728 & 0.044 \\
\hline & Primary School & 75 & 107 & & \\
\hline & Middle School & 50 & 59 & & \\
\hline & Technical Secondary / High School & 36 & 66 & & \\
\hline & College and Above & 36 & 56 & & \\
\hline \multirow{5}{*}{$\begin{array}{l}\text { Average monthly medical } \\
\text { expenses in the past six } \\
\text { months (CNY) }\end{array}$} & & & & 17.592 & 0.000 \\
\hline & $<2000$ & 103 & 193 & & \\
\hline & $2000 \sim$ & 37 & 34 & & \\
\hline & $5000 \sim$ & 28 & 24 & & \\
\hline & $10000 \sim$ & 37 & 29 & & \\
\hline \multirow{4}{*}{ Course of the disease (Year) } & & & & 7.931 & 0.005 \\
\hline & $<1$ & 56 & 103 & & \\
\hline & $1 \sim$ & 68 & 98 & & \\
\hline & $10 \sim$ & 81 & 79 & & \\
\hline \multirow{3}{*}{ Number of hospitalization } & & & & 7.067 & 0.008 \\
\hline & 1 & 45 & 92 & & \\
\hline & $2 \sim$ & 160 & 188 & & \\
\hline \multirow[t]{5}{*}{ Self-care ability } & & & & 70.461 & 0.000 \\
\hline & Completely Self-sufficient & 81 & 215 & & \\
\hline & Partial Self-sufficient & 91 & 52 & & \\
\hline & In Need of Great Help & 26 & 9 & & \\
\hline & Totally Dependent & 7 & 4 & & \\
\hline \multirow[t]{5}{*}{ Complications (Species) } & & & & 7.230 & 0.065 \\
\hline & 0 & 157 & 234 & & \\
\hline & 1 & 36 & 41 & & \\
\hline & 2 & 8 & 4 & & \\
\hline & 3 & 4 & 1 & & \\
\hline
\end{tabular}

In Table 3, some domains are statistical significance, those domains can influence depressive symptoms in patients with chronic diseases, such as education level, average monthly medical expenses, duration of illness, number of hospitalizations and self-care ability. In addition, the results which include the average monthly medical expenses, course of disease, self-care ability and education level were statistical significance.

Table 3. Logistic regression analysis of influencing factors for depressive symptoms in patients with chronic diseases ( $n=485$ )

\begin{tabular}{|c|c|c|c|c|c|}
\hline Variables & Partial regression coefficient & Standard error & Wald & $P$-value & $O R$ value $(95 \% \mathrm{CI})$ \\
\hline Average monthly medical expenses & 0.245 & 0.095 & 7.197 & 0.007 & $1.289(1.071-1.551)$ \\
\hline Course of disease & 0.347 & 0.123 & 7.933 & 0.005 & $1.415(1.111-1.801)$ \\
\hline Self-care ability & 1.066 & 0.152 & 49.104 & $<0.001$ & $2.905(2.156-3.914)$ \\
\hline Education level & -0.179 & 0.091 & 3.859 & 0.049 & $0.836(0.700-1.000)$ \\
\hline
\end{tabular}

\section{Discussion}

Base on some reports, the severity of depression has been measured by counting how many depression symptoms are present for a given individual, but it has been argued that such an aggregate measure might not adequately portray the complexity of depression $[5,12]$. In an additional method, researchers examine the network structure and dynamics of special depression symptoms, the connected situation was the 
judgment standard in result. For instance, a denser or more tightly knit network of symptoms, it may indicate a higher risk for developing depression [13]. Furthermore, a more strongly connected network structure of depression symptoms has also been shown to characterize persistent depression compared to more transient depression [14]. In addition, patients with coexisting depressed mood and chronic disease showed significantly more frequent doctor visits compared to those with only depressed mood or chronic disease [15]. Another population-based study with 1050 individuals aged 65-85 also indicated that depressive patients with multimorbid chronic disease expended higher total health care costs per six-month period compared to non-depressive patients [16].

Base on our research result, this investigation showed that the incidence of depressive symptoms in patients with 6 kinds of chronic diseases is $42.3 \%$, that the result is close to the results of similar studies [13]. The depression situations of participants are 101 cases with mild depression (49.3\%), 62 cases with moderate depression (30.2\%), and 42 cases with major depression (20.5\%). In total depression situation, patients with moderate and above depressive symptoms account for $50.7 \%$ of all the depressive patients included in the study, indicating that depressive symptoms are common in these patients and the degree is relatively severe. According to previous studies, a total of 104 patients included in this investigation with moderate and severe depressive symptoms can be diagnosed as a depressive disorder. The research result indicate chronic disease as a life stress event can lead to dysfunction in the hypothalamic-pituitary-adrenal axis, accompanied by depressive symptoms. The mutual influence of somatic diseases and depressive emotion leads to the increase of psychological pain, the decrease in quality of life and the increase of death rate in patients with chronic diseases [17].

In conclusion, the results of this study showed that the course of the disease is positively correlated with the incidence of depressive symptoms, the longer the course the higher the incidence of depression, consistent with similar studies. The study also found that the worse the self-care ability, the higher the incidence of depression, consistent with the results of similar studies. Patients with self-care deficits rely on the care of their caregivers, which places a great burden on individuals and families.

\section{Funding}

The research study was financially supported by the funding as awarded by the Guangdong Nursing Association (Item no.: gdhl xueh2019zx 303).

\section{References}

[1] Mcelroy E, Napoleone E, Wolpert M, Patalay P. EClinical Medicine structure and connectivity of depressive symptom networks corresponding to early treatment response. E Clinical Medicine. 2019; 8: 29-36.

[2] Brody DJ, Pratt LA, Hughes JP. Prevalence of Depression Among Adults Aged20 and over: United States, 2013-2016. NCHS Data Brief. 2018.
[3] Greenberg PE, Fournier AA, Sisitsky T, Pike CT, Kessler RC. The economic burden of adults with major depressive disorder in the United States (2005 and2010). J. Clin. Psychiatr. 2015.

[4] Epskamp S, Borsboom D, Fried EI. Estimating psychological networks and their accuracy: a tutorial paper. Behav. Res. Methods. 2018; 50 (1): 195-212.

[5] Van Borkulo C, Boschloo L, Borsboom D, Penninx BWJH, Lourens JW, Schoevers RA. Association of symptom network structure with the course of longitudinal depression. JAMA Psychiatry. 2015; 72 (12): 1219-1226.

[6] World Health Organization/ Newsroom. Non-communicable diseases. 2018. retrieved from. https://www.who.int/zh/news-room/fact-sheets/detail/noncom municable-diseases.

[7] Hongjin Li, Song Ge, Brian Greene, et al. Depression in the context of chronic diseases in the United States and China. International Journal of Nursing Sciences. 2019; 6 (1).

[8] Birk JL, Kronish IM, Moise N, et al. Depression and Multimorbidity: Considering Temporal Characteristics of the Associations Between Depression and Multiple Chronic Diseases. Health Psychology. 2019; 38 (9): 802-811.

[9] Khaledi M, Haghighatdoost F, Feizi A, et al. The prevalence of comorbid depression in patients with type 2 diabetes: an updated systematic review and meta-analysis on huge number of observational studies. Acta Diabetologica. 2019: 1-20.

[10] Milner A, Krnjacki L, Butterworth P, et al. The role of social support in protecting mental health when employed and unemployed: A longitudinal fixed-effects analysis using 12 annual waves of the HILDA cohort. Social Science \& Medicine. 2016; 153: 20-26.

[11] Fried EI, Nesse RM. Depressionsum-scores don'tadd up: whyanalyzingspecific depression symptoms is essential. BMC Med. 2015; 13 (1): 1-11.

[12] Cramer OJ, Borkulo Van CD, Giltay EJ, Maas Van Der HLJ, Kendler KS, Scheffer M, et al. Major depression as a complex dynamic system. 2016; 1-20.

[13] Yasaitis LC, Berkman LF, Chandra A. Comparison of self-reported and medicare claims-identified acute myocardial infarction. Circulation. 2015; 131 (17): 1477-1485.

[14] Lacruz ME, Emeny RT, Haefner S, Zimmermann AK, Linkohr B, Holle R, Ladwig KH. Relation between depressed mood, somatic comorbidities and health service utilisation in older adults: results from the KORA-Age study. Age Ageing. 2017; 41: 183-190.

[15] Chang-Quan H, Xue-Mei Z, Bi-Rong D, Zhen-Chan L, Ji-Rong Y, Qing-Xiu L. Health status and risk for depression among the elderly: a meta-analysis of published literature. Age Ageing. 2018; 39: 23-30.

[16] Jaakko A, Kia G, Mika K, Markus J. Connectivity of depression symptoms before and after diagnosis of a chronic disease: A network analysis in the U.S. Health and Retirement Study. Journal of Affective Disorders. 2020; 266: 230-234.

[17] Dickens C. Depression in people with coronary heart disease: prognostic sig-nificance and mechanisms. Curr. Cardiol. Rep. 2015; 17: 83 . 